\title{
Expandiendo el espectro mutacional en pacientes chilenos con fibrosis quística
}

\author{
MARCOS VÁSQUEZ D.*,\#, GUILLERMO LAY-SON R.*,\#, FELIPE VIAL U.*, \\ MARÍA LINA BOZA C.**, ILSE CONTRERAS E.***, JAIME LOZANO C.****, \\ DANIEL ZENTENO A.***** y MARÍA GABRIELA REPETTO L.*
}

\section{Expanding the CFTR mutation spectrum in Chilean patients with cystic fibrosis}

Introduction: Cystic Fibrosis (CF) is an autosomal recessive disease and affects 1 in 8000-9000 newborns in Chile. More than 1,800 different mutations have been identified in CFTR gene. The available molecular diagnosis analyzes the 36 most frequent mutations in Caucasian population, with an overall detection rate of $80-85 \%$, but with a much lower detection rate in Chilean patients of $42 \%$. To analyze which other mutations are present in Chilean patients, we conducted an extensive analysis by direct DNA sequencing of coding sequences of the CFTR gene. Methods: Forty eight Chilean patients with clinical diagnosis of CF and one mutated allele in the CFTR gene identified, were studied by direct sequence analysis of exons 6, 7, 14, 19 and 20 of the CFTR gene. Results: We found 3 different mutations in 14 cases that had not been previously identified in Chilean patients. Four patients have a deletion of two nucleotides (c.2462_2463delGT/p.Ser821ArgfsX4) in exon 14, which is predicted to cause a frameshift and a premature stop codon. Eight patients have c.3196C $>$ T mutation in the exon 20 and 2 cases has c.3039delC mutation in the exon 19. Both mutations had been previously described in other populations. Discussion: The identification of these mutations has notably increased the detection rate in our patients. Adapting the molecular diagnosis method by including these three mutations should increase the CF detection rate in Chilean patients. This analysis will improve CF diagnosis and allow an adequate genetic counseling to the families.

Key words: CFTR, Cystic fibrosis, mutation, sequence analysis, Chile.

\section{Resumen}

Introducción: La fibrosis quística (FQ) es una enfermedad con herencia autosómica recesiva, que presenta una incidencia de 1 en 8.000 a 9.000 recién nacidos en Chile. A la fecha se han descrito más de 1.800 mutaciones diferentes en el gen CFTR. El diagnóstico molecular disponible consiste en el análisis de las 36 mutaciones presentes con mayor frecuencia en población caucásica, donde se describe una tasa de detección de un 85\%. Sin embargo, en Chile el rendimiento corresponde a un 42\%. Por esta razón, hemos iniciado un análisis sistemático en la región codificante del gen CFTR con el fin de identificar los restantes alelos en pacientes chilenos con FQ. Métodos: Análisis por secuenciación de los exones 6,7,14,19 y 20, en 48 pacientes chilenos del Programa Nacional de FQ. Se incluyeron pacientes con criterios clínicos y de laboratorio de FQ, y con sólo una mutación identificada en el panel de 36 mutaciones. Resultados: Se identificaron 3 mutaciones diferentes que no se analizan en el panel de diagnóstico molecular y que no habían sido reportadas en pacientes chilenos, totalizando 14 casos. Cuatro casos corresponden a una nueva mutación en el exón 14, que produce un corrimiento en el marco de lectura y un codón de término prematuro (c.2462_2463delGT/p.Ser821ArgfsX4). Ocho casos presentan la mutación c.3196C> T en el exón 20, mientras que en 2 casos se encontró la mutación c.3039delC en el exón 19. Ambas mutaciones han sido descritas previamente en otras poblaciones.

\footnotetext{
* $\quad$ Centro de Genética Humana, Facultad de Medicina, Clínica Alemana-Universidad del Desarrollo. Santiago-Chile.

** Hospital San Borja-Arriarán, Santiago.

*** Hospital Padre Hurtado, San Ramón, Santiago.

**** Clínica Santa María, Providencia, Santiago.

*****Hospital Dr. Guillermo Grant Benavente, Concepción.

\# Ambos autores contribuyeron por igual en este trabajo.
} 
Discusión: La identificación de estas mutaciones ha incrementado notablemente la tasa de detección obtenida en nuestros pacientes. Esto crea la necesidad de adaptar el análisis molecular inicial en pacientes chilenos con FQ, redundando en un diagnóstico de certeza en gran parte de los casos y permitiendo un adecuado asesoramiento genético para las familias.

Palabras clave: CFTR, Fibrosis quística, mutaciones, análisis de secuencia, Chile.

\section{Introducción}

La fibrosis quística (FQ) es una enfermedad con herencia autosómica recesiva, que presenta una incidencia de 1 en 8.000 a 9.000 recién nacidos en Chile ${ }^{1}$ y se caracteriza principalmente por infecciones pulmonares recurrentes, insuficiencia pancreática y test de sudor alterado. La FQ es causada por mutaciones en el gen CFTR que tiene un tamaño de 250 kilobases (kb) organizado en 27 exones. A la fecha, se han descrito más de 1.800 mutaciones distintas a lo largo de todo el gen ${ }^{2}$, donde la mutación p.Phe508del es la más prevalente a nivel mundial. El diagnóstico molecular actualmente disponible en nuestro país consiste en el análisis de las 36 mutaciones más frecuentes en población caucásica, que tiene una tasa de detección de alrededor de un 90\% en caucásicos ${ }^{3}$, pero sólo un $42 \%$ en la población chilena ${ }^{4}$. Esta baja sensibilidad sugiere que en nuestra población predominan mutaciones ya descritas pero que no están incluidas en el panel, o bien existen mutaciones "locales" no descritas previamente. Por esta razón, hemos iniciado un análisis sistemático en la región codificante del gen CFTR, con el fin de identificar los restantes alelos en pacientes chilenos con FQ.

\section{Métodos}

\section{Selección de pacientes}

Cuarenta y ocho pacientes pertenecientes al programa nacional de Fibrosis Quística (PNFQ) con diagnóstico clínico de la enfermedad, test de sudor alterado y una mutación identificada con el panel de 36 mutaciones, fueron incluidos en el estudio. Los pacientes del PNFQ firmaron un consentimiento informado, en el cual autorizan el análisis y el almacenaje de su muestra para el análisis en búsqueda de mutaciones en CFTR. Las mutaciones identificadas por secuenciación se evaluaron luego en 42 pacientes del PNFQ con test de sudor alterado, pero sin mutaciones detectadas con el panel de 36 mutaciones.

\section{Preparación de ADN}

El ADN genómico fue preparado de linfocitos de sangre periférica utilizando el ensayo
AxyPrep $^{\mathrm{TM}}$ Blood Genomic DNA Miniprep de Axygen Biosciences (Union City, California).

\section{Amplificación y secuenciación}

Los exones 6, 7, 14, 19 y 20 fueron amplificados mediante la reacción en cadena de la polimerasa (PCR) utilizando oligonucleótidos específicos y complementarios a los bordes de cada exón (Tabla 1). El tamaño de los productos de PCR fue confirmado mediante electroforesis en geles de agarosa, purificados y secuenciados en Macrogen Inc. (Seoul, Corea). El análisis de secuencias fue realizado con el programa SeqScape ${ }^{\circledR}$ (Applied Biosystems).

\section{Análisis de las mutaciones identificadas}

El producto de PCR de un paciente con la mutación c.2462_2463delGT fue ligado en el vector pGEM ${ }^{\circledR}-T$ easy de Promega (Madison, USA) y luego se transformaron células competentes, según instrucciones incluidas en el ensayo. Las colonias positivas fueron secuenciadas con el oligonucleótido CFTR_13R en Macrogen Inc (Seoul, Corea).

La confirmación de la mutación c.2462_2463 delGT se realizó mediante una PCR utilizando los oligonucleótidos CFTR_13F HphI y CFTR_13R HphI (Tabla 1) seguido de digestión enzimática con la enzima HphI de New England BioLabs (Ipswich, Massachusetts, USA) y visualización en geles de agarosa al 3\% en tampón TBE.

La mutación c.3196C > T fue confirmada mediante una PCR utilizando los oligonucleótidos CFTR_20F y CFTR_20R TaqI (Tabla 1) seguido de digestión enzimática con la enzima TaqI de Fermentas (Burlington, Canadá) y visualización en geles de agarosa al 3\% en tampón TBE.

\section{Resultados}

\section{Secuenciación de ADN}

En cuatro pacientes en los que previamente se había detectado un alelo con la mutación p.Phe508del, se identificó una mutación denominada c.2462_2463delGT en el dominio regulatorio de la proteína CFTR (exón 14). Esta mutación se caracteriza por la deleción de dos nucleótidos GT lo que provoca un corrimiento en el marco de lectura y genera un codón de término prematuro (p.Ser821ArgfsX4) (Figura 1). 
Tabla 1. Secuencias de los oligonucleótidos utilizados para secuenciación y análisis de las mutaciones

\begin{tabular}{|cll|}
\hline Exón & Oligonucleótidos & 'secuencia \\
\hline 6 & CFTR_6F & 5'-TAGGTTATCAATTTTTGCGAAGTGTTTGATCATATAAGCTCC-3' \\
& CFTR_6R & 5'-CAGTCCTGGTTTTACTAAAGTGGGC-3' \\
\hline 7 & CFTR_7F & 5'-CTATTTGAGATTCTTTAGATAATAAAATAATGCCCATCTG-3' \\
& CFTR_7R & 5'-CAGTCCTGGTTTTACTAAAGTGGGC-3' \\
\hline 14 & CFTR_14F & 5'-CAAAATGCTAAAATACGAGACATATTGC-3' \\
& CFTR_14R & 5'-GAGTTTTAAGATACACCTTATCCTAATCC-3' \\
& CFTR_14F HphI & 5'-CAAGAAACTGGCTTGGAAATAGG-3' \\
& CFTR_14R HphI & 5'-CTCAATTGCATTCTGTGGGATG-3' \\
& CFTR_19F & 5'-AGTTTGAGGTGTTTAAAGTATGC-3' \\
& CFTR_19R & 5'-TAAAGAATCTCAAATAGCTCTTATAGC-3' \\
& CFTR_20F & 5'-GAAATTACATTTTGTGTTTATGTTATTTGC-3' \\
& CFTR_20F & 5'-CAAAAATTGATAACCTATAGAATGCAGC-3' \\
& CFTR_20R TaqI & 5'-AAAGTAAGGCTGCCGTCCGAAGGCTC-3' \\
\hline
\end{tabular}

A
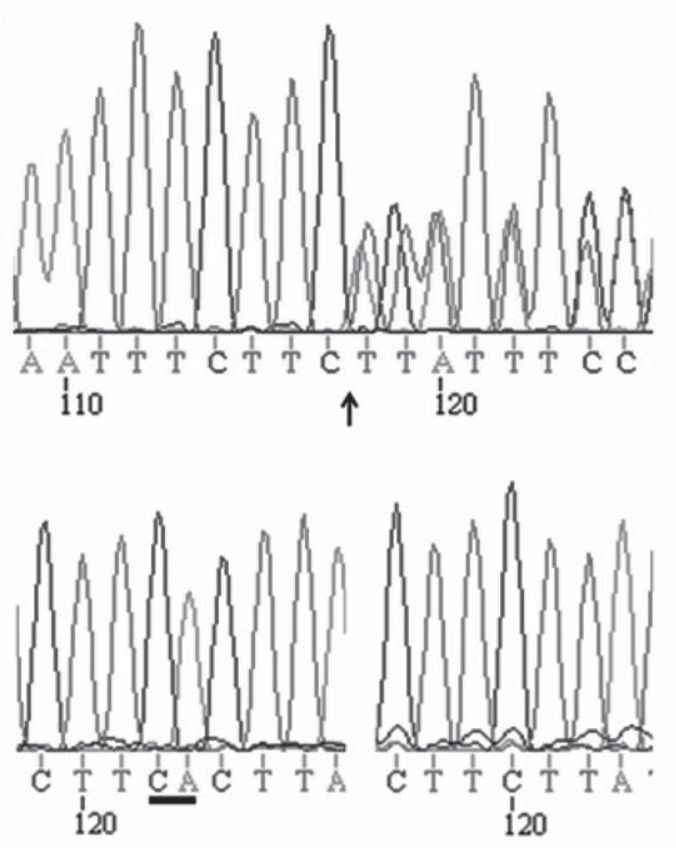

B AGT.GAA.GAA.ATT.AAC

Ser Glu Glu lle Asn

$\begin{array}{lllll}821 & 822 & 823 & 824 & 825\end{array}$

AGA.AGA.AAT·TAA.C

Arg Arg Asn Stop

821822823824

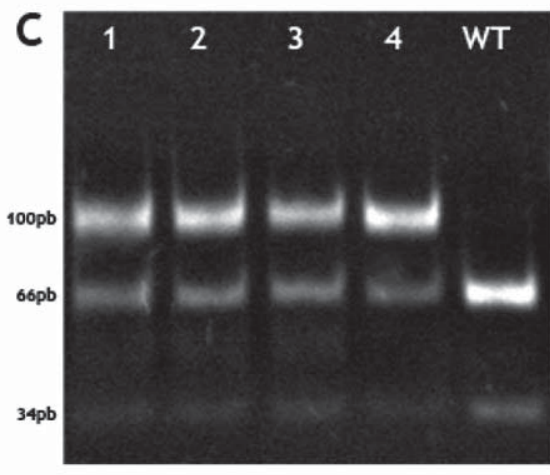

Figura 1. A) Resultado de secuenciación del exón 14 de CFTR. El electroferograma superior describe una mutación heterocigota para c.2462_2463delGT (p.Ser821ArgfsX4) en un paciente. Se produce superposición de los picos a partir del lugar donde ocurre la deleción (flecha). Este resultado fue obtenido al secuenciar con el oligonucleótido CFTR_14R. El electroferograma inferior corresponde a la secuencia del alelo normal (izquierda) y con la mutación (derecha) de un paciente. Los alelos fueron separados por clonamiento en el vector PGEM®-T Easy. Los nucleótidos deletados están destacados con una línea negra inferior. B) Esquema que ilustra el efecto de la deleción c.2462_2463delGT (gris) en el exón 821. Se produce un corrimiento del marco de lectura y un codón de término prematuro. La secuencia normal corresponde al esquema superior y la mutada al esquema inferior. C) Confirmación de la mutación en los demás pacientes mediante una PCR y digestión con la enzima HphI. La secuencia normal es reconocida por la enzima y produce fragmentos de 66 y 34 pb, mientras que la secuencia mutante no es cortada y presenta el tamaño del amplicón original (100 pb). Carriles 1 a 4 corresponden a pacientes con la mutación en estado heterocigoto. Carril 5 representa a un paciente sin la mutación (WT). 
En el exón 20, fue encontrada la mutación c.3196C > T en 8 pacientes. Esta mutación provoca el cambio del aminoácido Arginina por Cisteína (p.Arg1066Cys) en la proteína CFTR.

Por último, en dos casos se identificó la mutación c.3039delC en el exón 19 de CFTR. Esta mutación provoca la deleción del nucleótido de citosina generando un corrimiento en el marco de lectura y un codón de término prematuro (p.Tyr1014ThrfsX9).

\section{Confirmación de las mutaciones}

Se realizó la separación del alelo con la mutación c.2462_2463delGT y del alelo normal mediante ligación en el vector de clonación pGEM ${ }^{\circledR}-\mathrm{T}$ Easy. Posteriormente, se realizó la transformación de células competentes y se seleccionaron las colonias positivas. Diez de estas colonias fueron sometidas a una PCR y luego fueron secuenciadas. El análisis de las secuencias permite determinar con precisión los nucleótidos deletados (Figura 1A). Los cuatro pacientes con la mutación c.2462_2463delGT, fueron confirmados mediante una reacción de PCR seguida de digestión con la enzima HphI (Figura 1C). Además, se estudiaron a los padres de uno de los pacientes, siendo uno de ellos portador de la mutación p.Phe508del y el otro portador de la nueva mutación. Estos resultados confirman que ambas mutaciones se encuentran en cromosomas diferentes (trans) en el paciente afectado con FQ. Adicionalmente, se analizaron 42 pacientes con diagnóstico clínico de FQ, pero sin ninguna mutación previamente identificada en el ensayo de 36 mutaciones. En este análisis no encontramos nuevos pacientes con esta mutación (dato no mostrado).

Los 8 pacientes con la mutación c.3196C>T fueron confirmados por PCR y digestión con la enzima TaqI. Al igual que la mutación c.2462_2463delGT, se realizó este análisis en los 42 pacientes con diagnóstico clínico de FQ pero sin hallazgos en el panel de 36 mutaciones. En este análisis no encontramos nuevos pacientes con esta mutación (dato no mostrado).

\section{Discusión}

La identificación de estas mutaciones en nuestro grupo de pacientes es un aporte en la caracterización genética de la enfermedad en Chile. En este análisis se inició secuenciando algunos exones, que fueron seleccionados por la gran proporción de mutaciones que han sido reportados en ellos². Se decidió estudiar pacientes que tenían una clínica compatible con FQ, un test de sudor alterado y ya se había detectado una mutación con el panel de 36 mutaciones, debido a que en ese grupo existe una mayor posibilidad de encontrar el alelo restante ${ }^{5}$. Este hecho fue reafirmado, ya que al buscar en forma dirigida estas mutaciones en 42 individuos en quienes el panel de 36 mutaciones fue negativo.

La mutación en el exón 14 denominada c.2462_2463delGT/p.Ser821ArgfsX4, fue también recientemente identificada por un grupo de investigadores en Colombia ${ }^{2}$. Aunque aún no disponemos de estudios funcionales, esta mutación es altamente probable que sea patogénica ya que produce un corrimiento en el marco de lectura, y adicionalmente un codón de término prematuro, determinando una proteína truncada. Además, en uno de los pacientes se estudiaron ambos padres, determinándose que la mutación se encontraba en trans respecto al otro alelo.

La mutación c.3039delC en el exón 19 (nomenclatura antigua: 3171delC), ha sido reportada previamente en un paciente heterocigoto en Brasil $^{6} \mathrm{y}$ en un paciente heterocigoto en una población de origen hispánico en Estados Unidos ${ }^{7}$. Esta mutación también genera un desplazamiento del marco de lectura y un codón de término anticipado.

La mutación c.3196C> T del exón 20 también ha sido reportada en pacientes de origen hispánico ${ }^{2,8,9}$.

El hallazgo de las primeras dos mutaciones señaladas arriba apoya la hipótesis de mutaciones propias de Latinoamérica que sería importante verificar en otras poblaciones de nuestro continente.

Tomando en cuenta un total de 578 alelos (289 pacientes) analizados a la fecha, se ha podido detectar una mutación patogénica en 257 alelos de ellos, por lo que la tasa de detección alcanzada es de un 44,5\%. De hecho, hasta el momento estas 3 nuevas mutaciones pasan a ocupar el lugar 5 (c.3196C>T), 8 (c.2462_2463delGT) y 11 (c.3039delC) en frecuencia en pacientes chilenos. Considerando que con el actual panel molecular existe un bajo nivel de detección de los alelos causantes de FQ en Chile, se genera la necesidad de adaptar el protocolo utilizado a nuestra realidad local y regional. Las perspectivas a futuro es lograr secuenciar el resto de los 27 exones del gen CFTR en los pacientes del PNFQ. De esta manera se podrá alcanzar un mayor índice diagnóstico sobre todo en los casos en que es difícil confirmar o descartar la enfermedad, permitiendo establecer tempranamente las medidas terapéuticas necesarias, y entregar un adecuado asesoramiento genético para las familias. 


\section{Referencias bibliográficas}

1.- ASTUDILLO P. Fibrosis quística en Chile. Neumol Pediatr 2010; 5: 42-3.

2.- Cystic Fibrosis Mutation Database (CFMDB) [Internet]. 2011; Disponible en: http://www.genet.sickkids.on.ca/ cftr/StatisticsPage.html

3.- MOSKOWITZ S M, CHMIEL J F, STERNEN D L, CHENG E, CUTTING G R. CFTR-Related Disorders. 2001; [citado 2011 Sep 12] Disponible en: http://www. ncbi.nlm.nih.gov/books/NBK1250/

4.- LAY-SON G, PUGA A, ASTUDILLO P, REPETTO G M. Cystic fibrosis in Chilean patients: Analysis of 36 common CFTR gene mutations. J Cyst Fibros 2011; 10: 66-70.

5.- DEQUEKER E, STUHRMANN M, MORRIS M A, CASALS T, CASTELLANI C, CLAUSTRES M, et al. Best practice guidelines for molecular genetic diagnosis of cystic fibrosis and CFTR-related disorders-updated European recommendations. Eur J Hum Genet 2009; 17: 51-65.

6.- BERNARDINO A L, FERRI A, PASSOS-BUENO M R, KIM C E, NAKAIE C M, GOMES C E, et al. Molecular analysis in Brazilian cystic fibrosis patients reveals five novel mutations. Genet Test 2000; 4: 69-74.

7.- $\quad$ WONG L J, WANG J, ZHANG Y H, HSU E, HEIM R A, BOWMAN C M, et al. Improved detection of CFTR mutations in Southern California Hispanic CF patients. Hum Mutat 2001; 18: 296-307.

8.- LUZARDO G, AZNAREZ I, CRISPINO B, MIMBACAS A, MARTÍNEZ L, POGGIO R, et al. Cystic fibrosis in Uruguay. Genet Mol Res 2002; 1: 32-8.

9.- VISICH A, ZIELENSKI J, CASTAÑOS C, DIEZ G, GRENOVILLE M, SEGAL E, et al. Complete screening of the CFTR gene in Argentine cystic fibrosis patients. Clin Genet 2002; 61: 207-13.

Correspondencia a: Marcos Vásquez Durán

Bioquímico Centro de Genética Humana - Facultad de Medicina Clínica Alemana - Universidad del Desarrollo Av. Las Condes 12438. Lo Barnechea. Santiago-Chile. Fono: 2-3279263

E-mail: mvasquez@udd.cl 